\title{
Characterization of Electron Beam Irradiated and Alkali Treated Henequen (Agave Fourcroydes) Fibers Using SEM, TEM, EDS and CP/MAS C ${ }^{13}$ NMR
}

\author{
R.L. Schalek*, Seong Ok Han, Y.J. Yoo**, Y.S. Ahn**, and M.H. Han** \\ *Harvard University, Center for Imaging and Mesoscale Structures, Cambridge, MA 02138 \\ **Korean Institute of Energy Research, Yusong, Taejon 305-600
}

Bio-based composite materials derived from renewable resources constitute recyclable and sustainable materials. The fabrication of natural fiber based composites typically requires a fibersurface treatment to optimize composite properties [1]. Natural fibers have varying content of cellulose, hemicellulose and lignin with the hydrophilic nature of cellulosic fibers preventing adhesion and dispersion in hydrophobic matrices [2]. Improved fiber-matrix adhesion and dispersion results from surface chemistry altering treatments. Although not an intentional fiber treatment, the use of electron-beam composite curing technology necessarily exposes the fiber to high doses of ebeam irradiation [3]. In analogy to chemical methods of fiber treatments, ebeam curing potentially alters the fiber surface morphology, crystallinity and chemistry.

In this work the morphological, chemical and crystalline properties of the henequen (Agave Fourcroydes) leaf fiber treated using electron beam irradiation and alkali solution treatments were examined using SEM, TEM, EDS, and NMR. The electron beam irradiation doses ranged from 50 to $1000 \mathrm{kGy}$, while the alkali treatments consisted of 1-hour soak in $2 \%, 5 \%$, and $10 \%$ solutions. The SEM micrographs in Figure 1 compares the surface morphology of raw, ebeam and alkali treated fibers. The ebeam irradiations darken and embrittle the fibers without noticeable morphological surface changes. EDS surface analysis indicated both treated fibers were highly oxidized. In addition, the SEM investigation of the alkali treated fibers shows remnants of the surface treatments. Figure 2 shows the fiber cross sections of the fibers. The raw and ebeam irradiated fibers are similarly consistent with strong cell walls (no cellular content), while the alkali treatment significantly degrades the inner cell wall components and only the outer cell wall remains. In contrast, the alkali treatments caused increased fibrillar separation and cell wall collapse with increasing concentrations.

An NMR study of the alkali treated fibers indicates a loss of amorphous cellulose (fibril surface), while the crystalline cellulose peak shifts and increases in intensity. In contrast, the ebeam treated fibers, show no change in the amorphous peak intensity and the crystalline cellulose shows a peak shift indicating the occurrence of free radicals and/or bond cleavage. Additional information concerning the cellulose and lignin content will be determined using immunogold labeling and TEM analysis.

References

[1] G. Canche-Escamilla et al., Composites: Part A, 30 (1999) 349.

[2] A.K. Mohanty et al., Compos. Interfaces, 8, (2001) 313.

[3] H. Degrand et al., Radiation Physics and Chemistry, 68, (2003) 885.

[4] The authors would like to acknowledge EB-TECH Co., Taejon, Korea for use of the electron beam facilities and the Center for Imaging and Mesoscale Structures at Harvard Univerisity 

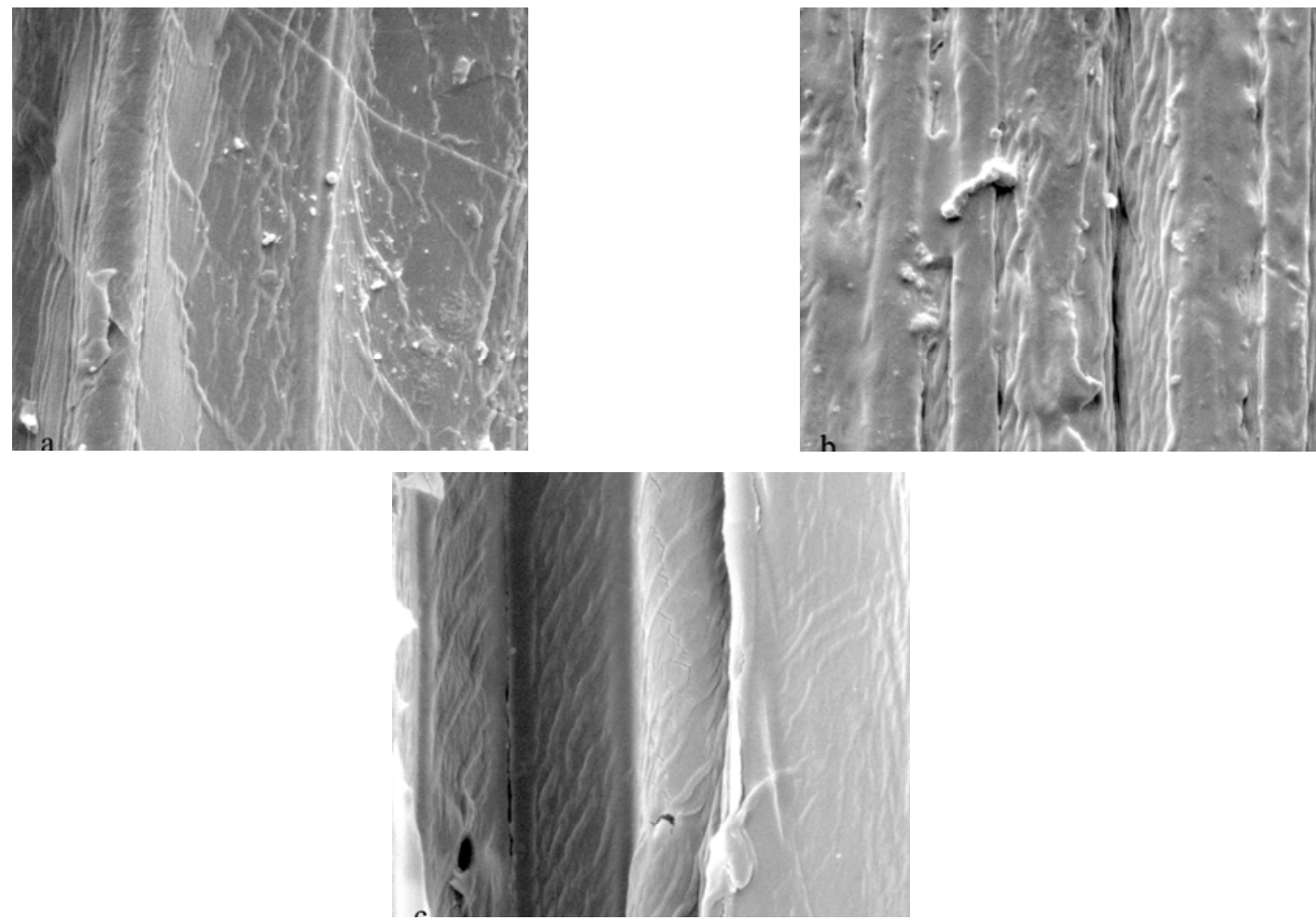

FIG. 1; SEM micrographs of the fiber surfaces for raw (a), 10\% alkali treated (b), and $500 \mathrm{kGy}$ treated henequen fibers (c) (HFW $=42.7$ microns).
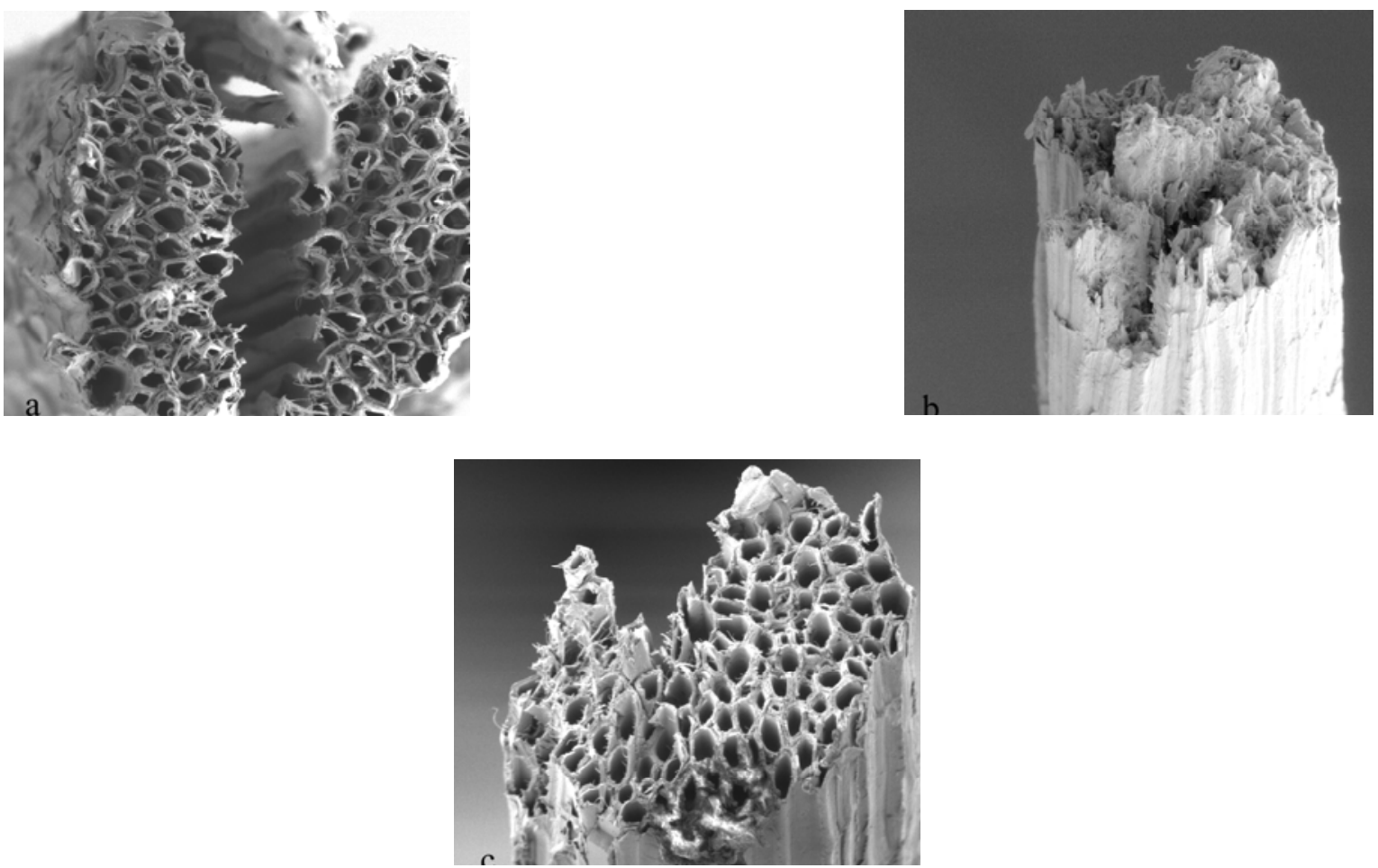

FIG. 2; SEM micrographs of the fiber cross sections for raw (a), 10\% alkali treated (b), and $500 \mathrm{kGy}$ treated henequen fibers (c) ( $\mathrm{HFW}=280$ microns). 